\title{
Mechanical properties of the hollow-wall graphene gyroid lattice
}

\author{
Kenichi Nakanishi, ${ }^{1}$ David Labonte, ${ }^{2}$ Tomasz Cebo, ${ }^{1}$ Vlad P. Veigang-Radulescu,,${ }^{1,3}$ Ye \\ Fan, ${ }^{1}$ Barry Brennan, ${ }^{3}$ Andrew J. Pollard, ${ }^{3}$ Stephan Hofmann, ${ }^{1,{ }^{*}}$ and Norman A. Fleck ${ }^{1, *}$ \\ ${ }^{1}$ Department of Engineering, University of Cambridge, Cambridge, United Kingdom CB2 \\ $1 \mathrm{PZ}$ \\ ${ }^{2}$ Department of Bioengineering, Imperial College London, South Kensington Campus, \\ United Kingdom, SW7 2AZ \\ ${ }^{3}$ National Physical Laboratory, Hampton Road, Teddington, Middlesex TW11 OLW, \\ United Kingdom
}

\begin{abstract}
The macroscopic elastic modulus and yield strength of solid-wall nickel gyroids and hollow-wall graphene gyroids of cell size $60 \mathrm{~nm}$ are deduced from indentation tests on a thin coating of the gyroids, with suitable interpretation by finite element simulations. The solid-wall nickel gyroids are fabricated by the self-assembly of a triblock copolymer, followed by the chemical vapour deposition of a graphene film onto this catalytic template. The nano-indentation response of the gyroid-based coatings was measured using a Berkovich indenter. In order to interpret the indentation response, two sets of finite element simulations were performed: periodic cell calculations in order to deduce the effective macroscopic properties in terms of the relative density and cell wall properties of the lattice, and then indentation simulations of a continuum with the effective properties of the gyroid. Despite the knockdown in modulus and strength of the graphene gyroid lattice due to waviness of the layered cell walls, the structure remains remarkably strong due to nanoscale size effects.
\end{abstract}

\section{Introduction}

The macroscopic mechanical properties of cellular solids are related to their relative density and micro-architecture of the cell walls ${ }^{1}$. Recent progress in growth and process technologies allow for the manufacture of architectured cellular solids at the nanoscale ${ }^{2-4}$. Such nanolattices have exceptional mechanical properties such as a high yield strain for the case of metallic lattices ${ }^{5,6}$ and a high fracture strain for ceramic lattices ${ }^{4}$. They possess nearly constant specific stiffness at ultra-low density ${ }^{7}$, and the cell walls possess ultra-high effective strengths ${ }^{8,9}$. Nanolattices therefore expand material property space, as illustrated in the plot of strength versus density of Figure 1. 
These novel mechanical characteristics arise from both microstructural architecture and from nanoscale size effects on the strength of cell wall material.

Strength can increase dramatically with decreasing structural size primarily due to reduction in the number of defects ${ }^{10-12}$. Recall that the bulk strengths of metals and ceramics are orders of magnitudes below their theoretical limits due to the presence of imperfections within the bulk, such as dislocations, grain boundaries, cracks and voids. Consider, for example, the tensile strength of a strut of cross-sectional thickness $t$. If the strut is to exist, then it can only contain defects of dimension less than $t$. Consequently, small struts can only contain small defects. This 'smaller is stronger' characteristic has driven the development of nanolattices, in which the length scale of the lattice is reduced to a scale small enough to exploit size-dependent strengthening. Pyrolytically derived ceramic nanolattices ${ }^{13}$, atomic layer deposited hollow-beam ceramic nanolattices ${ }^{14}$, and the nickel double gyroid nanolattice ${ }^{9}$ all demonstrate such size effects.

The topology of a lattice material dictates the relationship between macroscopic properties, such as macroscopic Young's modulus $E$, and relative density $\bar{\rho}$. A number of theoretical models and observations support the scaling law $E=C \bar{\rho}^{n} E_{s}$ where $E_{s}$ is the Young's modulus of the solid cell wall material, $C$ is a geometric parameter and the exponent $n$ for a 3D lattice has a value of 1 or 2 for an ideal stretching- or bendingdominated behavior of the cell wall struts, respectively ${ }^{1}$. The value of $C$ is sensitive to the details of the micro-architecture. Porous biological materials possess architectures adapted to strong selective pressures in combination with size effect strengthening ${ }^{15}$. The gyroid lattice is one such natural structure ${ }^{16}$, and nanoscale single solid-wall gyroids ${ }^{17}$, inter-connected double solid-wall gyroids ${ }^{9}$, and hollow-wall gyroid topologies ${ }^{2}$ have been recently synthesised. These lattices possess a high surface area to volume ratio and a uniform cell and pore size, all of which are beneficial for material systems requiring functional nanostructures ${ }^{18}$.

Khaderi et al. ${ }^{19}$ used finite element multi-axial collapse simulations of an idealised slender beam model to predict the elastic-plastic response of the gyroid lattice, and deduced that the macroscopic properties are close to isotropic. The beam model of the ideal gyroid lattice deforms by beam stretching under macroscopic hydrostatic stress, with a bending-dominated elasto-plastic response occurring for all other loading states. Likewise, the effective elastic and plastic properties of the solid-wall double gyroid unit ${ }^{9}$ has been computed for continuum elements by unit cell finite element analysis. The predicted variations of elastic modulus $E$ and yield strength $\sigma_{y}$ with relative density $\bar{\rho}$ for both the beam model and solid-wall double gyroid were found to scale as $E \sim \bar{\rho}^{2} E_{S}$ and $\sigma_{y} \sim \bar{\rho}^{3 / 2} \sigma_{y s}$, consistent with bending-dominated behavior. Nanoindentation 
measurements of a fabricated nickel double gyroid lattice were used to calibrate an inverse finite element analysis, extracting the associated properties of the parent nickel $^{9}$. It was found that the predicted yield strength of the struts (5.7 GPa) approaches the theoretical strength of nickel, and this was explained by dislocation starvation in the struts of nanoscale dimension due to the attraction of dislocations to the free surfaces of the struts.

The mechanical properties of hollow-wall graphene gyroid lattices have also been predicted by simulations employing molecular dynamics (MD) or density functional theory (DFT). These models assume that the walls of the gyroid lattice are made from a single layer of graphene, and they predict exemplary electrical ${ }^{20}$ and mechanical ${ }^{21-23}$ properties. However, in practice, the cell walls of graphene gyroid foams and lattices comprise multiple layers of graphene ${ }^{2,24}$. The interplanar shear modulus $G_{S}$ and shear strength $\tau_{y s}$ of layered graphene are orders of magnitude less than their in-plane counterparts, owing to the weak interlayer van der Waals interactions ${ }^{25}$. Interlayer shear within wavy multilayered (10+ layers) graphene walls leads to a multiplicative knock down in the macroscopic properties of graphitic foams ${ }^{26}$; this deformation mechanism may also be active in few-layered ( $<10$ layers) hollow-wall graphene gyroid lattices.

Here, we report on the manufacture and measurement of the mechanical properties of a nickel gyroid and of a hollow-wall graphene gyroid lattice. These nanolattices contain stocky cell walls of thickness on the order of $15 \mathrm{~nm}$ and graphitic wall thicknesses of approximately $3 \mathrm{~nm}$. A combination of indentation tests and multi-scale finite element analysis are used to deduce the effective macroscopic properties of these gyroid-based materials in terms of the underlying cell wall properties.

\section{Experimental Protocol and Measurements}

\subsection{Manufacture of nano gyroid lattice layers}

Solid-wall nickel gyroid lattices and hollow-wall graphene gyroid lattices were manufactured in the form of a coating of thickness between $300 \mathrm{~nm}$ and $700 \mathrm{~nm}$ by block copolymer self-assembly, as described in detail elsewhere ${ }^{27}$; the graphene deposition methodology is based on that developed by Cebo et al. ${ }^{2}$ The production process and morphology of the resulting nano-gyroid lattices are sketched in Figure 2. The solid-wall nickel gyroid lattice was manufactured as a coating on a $350 \mathrm{~nm}$ thick layer of fluorine-doped tin oxide (FTO), which in turn sat on a borosilicate glass slide of thickness $2.2 \mathrm{~mm}$. The nickel lattice was obtained by electroplating a template in the 
form of a polymeric gyroidal layer; this polymeric lattice was prepared by self-assembly of a polyisoprene-block-polystyrene-block-polyethylene oxide (ISO) triblock copolymer. The main details are as follows.

Solid-wall nickel gyroid lattices: Block copolymer samples of selected thickness between $300 \mathrm{~nm}$ and $700 \mathrm{~nm}$ were prepared on the FTO-coated glass slide. The thickness of the copolymer coating was dictated by the spin speed during deposition. The samples were annealed in a vacuum oven at $180^{\circ} \mathrm{C}$ to encourage the self-assembly of the gyroidal structure then slowly cooled. The polyisoprene (PI) block of the ISO triblock copolymer was degraded by UV exposure and removed by dissolution in ethanol. Nickel was electroplated into the void left after PI removal. The remaining polymers were then removed by oxygen plasma etching. A hydrogen annealing process removed any trace of organic residues, resulting in a self-supporting lattice, henceforth referred to as the solid-wall nickel gyroid. The resulting solid-wall nickel gyroid has a unit cell size of $60 \mathrm{~nm}$ and a fill fraction of $40 \%$ (i.e. relative density $\bar{\rho}=0.40$ ), as confirmed through analysis of cross-sectional images obtained via scanning electron microscopy (SEM) (see supplementary section $\mathrm{SI}-1$ ).

Hollow-wall graphene gyroid lattices: The nickel gyroid was used as catalytic template for the deposition of few-layer graphene in a custom-built low-pressure chemical vapour deposition (CVD) reactor. Acetylene gas was introduced into the system at room temperature at a flow rate of $5 \mathrm{sccm}$ and a working pressure of $2.2 \times 10^{-3} \mathrm{mbar}$. Samples were heated for 5 minutes at $650^{\circ} \mathrm{C}$, reached by ramping the temperature at $50^{\circ} \mathrm{C} / \mathrm{min}$. Samples were then allowed to cool at a rate of $50^{\circ} \mathrm{C} / \mathrm{min}$ to ambient temperature, within the reactor. The samples were immersed in an etchant solution $(0.1 \mathrm{M}$ ammonium persulfate) for 24 hours in order to remove the nickel lattice and were then repeatedly rinsed in de-ionised (DI) water in order to obtain a freestanding graphitic lattice, henceforth referred to as the hollow-wall graphene gyroid. The hollow-wall graphene gyroid was stable during the etching and rinsing stages and remained adhered to the substrate.

\subsection{Characterisation of nano gyroid lattice layers}

Atomic force microscopy (AFM) micrographs and SEM images (2kV, 50pA) of the top surface of the solid-wall nickel gyroid coating are shown in Figure $3 a$ and $b$. AFM micrographs were taken in PeakForce tapping mode with Bruker MMP-11200-10 tips $(40 \mathrm{~N} / \mathrm{m}, 300 \mathrm{kHz}$, asymmetric tip) at a $0.5 \mathrm{~Hz}$ scan rate. A cleaved cross-section in Figure $3 \mathrm{c}$ displays the layered structure of the gyroid/FTO/glass. Cross-sections of the gyroid coatings were prepared using focused ion beam (FIB) milling (Figs. 3, 4) in order to determine the coating thickness. Long-range order is evident through a vertical slice of 
the gyroid coatings, and it is clear that the hollow-wall graphene gyroid inherits its topology from the nickel gyroid template. Similar images were used to measure the coating thickness at multiple locations for each sample; the coating thickness of each sample is 300,500 and $700 \mathrm{~nm}$, each with a standard deviation of $\pm 25 \mathrm{~nm}$. The gyroid coatings tested in this study have an arithmetic average roughness of $R_{a}=25 \mathrm{~nm}$, as measured by AFM across a $5 \mu \mathrm{m}$ by $5 \mu \mathrm{m}$ area.

In order to determine the level of graphitisation, Raman spectra were taken with a Renishaw inVia spectrometer using $532 \mathrm{~nm}$ excitation, see Fig. 5a. Characteristic D, G, and $2 \mathrm{D}$ peaks are observed, with a G:2D ratio and a broad 2D peak that are consistent with the growth of few-layer graphene gyroids ${ }^{2}$. A prominent D-band and red-shifted Gband are present, consistent with the presence of numerous small, disordered ${ }^{28}$, and strained ${ }^{29}$ graphene domains. These characteristics arise from the nanoscale growth surface and high local curvature of the nickel gyroid template ${ }^{2}$ used to prepare the graphene gyroids.

In order to confirm the complete removal of the internal nickel template from the freestanding graphene gyroid, time of flight-secondary ion mass spectrometry (ToFSIMS) depth profiling was performed in a ToF-SIMS IV instrument (ION-TOF GmbH, Muenster, Germany), at a vacuum pressure of $<5 \times 10^{-9} \mathrm{mbar}$, equipped with an argon gas cluster ion beam (GCIB) mounted to impact the sample at $45^{\circ}$ on a graphene gyroid coating, see Fig. 5b. Argon cluster sputtering (10 keV, $\mathrm{Ar}_{2500^{+}}$ions, $1.0 \mathrm{nA}$ ) over an area of $400 \mu \mathrm{m} \times 400 \mu \mathrm{m}$ was used to sputter across the entire hollow-wall graphene gyroid

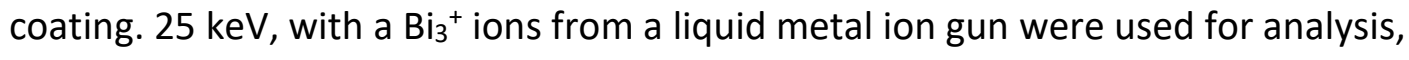
operating in interlaced mode at an ion current of $0.1 \mathrm{pA}$ and a cycle time of $200 \mu \mathrm{s}$, raster scanned randomly in an area of $150 \mu \times 150 \mu \mathrm{m}(256 \times 256$ pixel density $)$ in the center of the sputter crater to mitigate crater edge effects on the generated. Data processing was carried out by selecting relevant ion peaks in the ToF-SIMS spectra and monitoring their change in intensity over the course of the sputter profiling. The selected depth profiles were normalised to the total ion intensity using a point-to-point normalisation. The $\mathrm{C}^{+}$carbon signal is stable throughout the thickness of the gyroid layer but then reduces as a $\mathrm{SnO}^{+}$signal appears, indicating the bottom of the graphene gyroid coating, and the top of the FTO layer. The nickel $\mathrm{Ni}^{+}$signal is low throughout the scan, suggesting that the etching and rinsing steps have successfully removed the internal template. Other process contaminants such as $\mathrm{Na}^{+}$and $\mathrm{Ca}^{+}$were found at similarly negligible concentrations.

Transmission electron microscopy (TEM) samples were prepared from as-grown graphene gyroids by first removing the graphene gyroid coating from its substrate with a razor blade and then using wet transfer via water lift-off onto Au-supported holey 
carbon grids. Bright-field TEM images were taken at $80 \mathrm{kV}$ accelerating voltage in order to prevent beam damage to the material. The TEM images possess a lattice spacing of $0.33 \mathrm{~nm}$, consistent with few-layer graphene. The walls of the hollow-wall gyroid comprise 8-10 layers of graphene, see Fig. 5c, corresponding to a wall thickness of $3 \mathrm{~nm}$. Hence, the continuous surface of the fabricated graphene gyroid lattice can be considered as a foam with stocky hollow-wall struts, possessing a relative density of $\bar{\rho} \approx$ 0.14 and a unit cell size of $60 \mathrm{~nm}$.

\subsection{Indentation Measurement Protocol}

Nanoindentation tests were performed on sets of three thicknesses of nickel gyroid and graphene gyroid coatings, using a Hysitron Ub1 Nanoindenter system of depth resolution $0.04 \mathrm{~nm}$. The Berkovich tip had a tip radius of $50 \mathrm{~nm}$ and an included half angle of $65.35^{\circ}$, as measured from the central axis to a pyramidal flat, and was calibrated against a standard fused silica sample. The tip was sufficiently sharp to be pyramidal at an indentation depth $\delta \geq 50 \mathrm{~nm}$. Sixteen repeat indentation tests were carried out on each of the gyroid coatings. Samples were indented in a 4 by 4 array with a $50 \mu \mathrm{m}$ spacing in both lateral directions to ensure that the strain fields due to the indent did not interact with each other. For each test, the peak load was progressively increased up to the maximum load of $11 \mathrm{mN}$ for nickel and $1 \mathrm{mN}$ for the hollow-wall graphene gyroids. Twelve partial load/unload cycles were performed per test. Each loading and unloading segment were of $2 \mathrm{~s}$ duration, with a $2 \mathrm{~s}$ hold time between each segment. During each unloading cycle, the load was reduced to $50 \%$ of the prior load. The modulus and hardness were extracted from these measurements using the standard Oliver and $\mathrm{Pharr}^{30}$ procedure. The detailed assumptions of this analysis are outlined in the supplementary information section SI-2.

\subsection{Measured properties of the gyroid coatings}

Fig. 6 shows SEM micrographs and surface profiles of the coatings post-indentation. For the solid-wall nickel gyroid coatings, indentation occurs by plastic deformation of the gyroid lattice with limited elastic recovery of the indent (see Fig. 6a). In contrast, for the hollow-wall graphene gyroid lattice, significant elastic recovery occurs; for example indentation to $\delta_{\max } / h \approx 0.79$ leads to a residual indent of $\delta_{f} / h \approx 0.13$. SEM images of the hollow-wall graphene gyroid after indentation show minimal plastic deformation and damage (Fig. 6c). The level of elastic recovery remained high $\left(\delta_{f} / h \approx 0.39\right)$ even after indentation to $\delta_{\max } / h \approx 0.99$. It is emphasised that deep indents lead to plastic deformation of the underlying FTO layer, thereby reducing the relative degree of elastic recovery of the indent. 
The measured values of unloading modulus $\mathrm{E}_{\mathrm{R}}$ and of hardness $H$ are plotted in Fig. 7 as a function of the normalised indentation depth $\delta / h$. The hardness $H$ is approximately independent of indent depth $\delta$, for $0.2<\delta / h<0.5$. At high values of indent depth $(\delta / h>0.5), H$ increases with increasing $\delta / h$ due to indentation of the substrate. The largest scatter in the measured values of $E_{R}$ and of $H$ occur for the thinnest gyroid coatings, due to the finite roughness of the FTO layer relative to the layer thickness $h$.

\section{Numerical simulations of the indentation of the solid-wall and hollow-wall gyroid lattice}

The Young's modulus and uniaxial compressive yield strength of the cell wall material in the solid-wall gyroid and in the hollow-wall gyroid cannot be determined directly from the nanoindentation measurements reported above. In this section, a multi-scale numerical analysis is utilised to extract the effective mechanical properties of the gyroid lattice and parent materials, for both the solid-wall nickel and hollow-wall graphene cases. Khaderi et al. ${ }^{19}$ have previously derived power-law scaling relations for the elastic constants of the solid-wall gyroid lattice through numerical simulation of an idealised slender-beam model of the gyroid lattice. However, at high relative densities, the struts of the gyroid lattice become stocky and will vary in size and cross-sectional shape along their length. Consequently, the slender beam approximation underestimates the modulus and strength of solid-wall gyroid lattices when $\bar{\rho}$ exceeds 0.15 . Analysis of the double gyroid lattice, which comprise two interpenetrating single gyroids of opposite chirality, has also been previously performed up to a limit of each single gyroid possessing a relative density of $\bar{\rho}=0.19$, see Khaderi et al. ${ }^{9}$. The solid-wall gyroid tested herein possesses a relative density $\bar{\rho}=0.40$, motivating the numerical simulation of solid-wall gyroid unit cells of relative density $\bar{\rho}$ up to 0.5 . A comparative plot of the effective properties of the solid-wall single gyroid calculated herein against the slender beam model ${ }^{19}$ and double gyroid model ${ }^{9}$ can be found in Figure SI- 2 .

\subsection{Effective properties of the solid-wall and hollow-wall gyroid}

The unit cells of the solid-wall gyroid and hollow-wall gyroid are shown in Figure 9. These unit cells are constructed using the approximation to the single gyroid morphology as proposed by Lambert et al. ${ }^{31}$ In this approximation, the surface of a single gyroid is represented by a function $F(x, y, z)-t_{0}=0$, where

$$
F \equiv \sin (2 \pi x) \cos (2 \pi y)+\sin (2 \pi y) \cos (2 \pi z)+\sin (2 \pi z) \cos (2 \pi z)
$$

in terms of the Cartesian coordinates $(x, y, z)$ aligned with the cubic directions of the gyroid lattice, as shown in Fig. 9 . The scaling parameter $t_{0}$ controls the distance of the 
surface from the $t_{0}=0$ surface, and hence determines the relative density of the gyroid lattice. The solid-wall gyroid is constructed by infilling the space $F-t_{0} \geq 0$ to obtain the volume shown in Figure 9a. The hollow-wall gyroids were based on the templated surface growth of graphene, and hence had a fixed value of $t_{0}$ such that the internal surface was equal to that of a solid-wall gyroid of relative density $\bar{\rho}=0.4$. The hollowwall gyroid was constructed by adopting an additional second scaling parameter $t_{1}$ and by infilling the space between two gyroid surfaces $F-t_{0}=0$ and $F-t_{1}=0$, to obtain the hollow-wall gyroid, as shown in Figure 9b.

Periodic cell finite element (FE) calculations were performed on each of the constructed volumes to determine the macroscopic, effective elastic and plastic properties using the commercial finite element package ABAQUS. The gyroid geometry was meshed using uniform 4-noded linear tetrahedral elements (C3D4 in ABAQUS notation). As the thickness to diameter ratio of each of the hollow-wall gyroids simulated herein exceeded 0.03 , shell elements were not used ${ }^{32}$. The mesh density was chosen such that a halving of the mesh size results in less than $1 \%$ change in the predicted macroscopic modulus or strength of the lattice, which required approximately $3 \times 10^{5}$ elements. The cell wall material was treated as an isotropic elastic, ideally plastic solid in accordance with $\mathrm{J} 2$ plasticity theory, with Young's modulus $E_{S}$, Poisson ratio $v_{S}$ and yield strength $\sigma_{S}$. We assume that $v_{S}=0.3$ and the yield strain $\epsilon_{S}=\sigma_{S} / E_{S}$ equals 0.03 .

The unit cell is subjected to periodic boundary conditions, such that every pair of nodes on two opposing faces of the unit cell was linked through linear constraint equations for each degree of freedom ${ }^{33}$. Reference nodes were linked to nodes of an entire face and used to impose boundary displacements as well as to extract the reaction forces. Simulations were then conducted to determine the three independent elastic constants of the double gyroid. The stress at $0.2 \%$ offset plastic strain was taken as the yield strength $\sigma_{S}$. The macroscopic Poisson ratio $v$ was calculated from the initial elastic strain increment. The plastic Poisson's ratio, $v_{P}$ was determined from the incremental Poisson's ratio during plastic flow. Details of the homogenisation method and the extraction of effective mechanical properties are given in the supplementary information section SI-4.

The relative density of both unit cells were varied by suitable selection of the scaling parameters $t_{0}$ and $t_{1}$. The predicted macroscopic elastic properties and macroscopic yield strength are plotted as a function of relative density $\bar{\rho}$ in Fig. 10. Curve fitting of these predictions for the solid-wall gyroid lattice (as denoted by the superscript $\bullet$ ) provides the following scaling relationships for $0.1<\bar{\rho}<0.5$ : 


$$
\begin{aligned}
& \frac{E^{\bullet}}{E_{S}}=0.90 \bar{\rho}^{2.11} \\
& \frac{G^{\bullet}}{E_{S}}=0.38 \bar{\rho}^{1.81} \\
& \frac{\sigma_{Y S}^{\bullet}}{\sigma_{S}}=0.48 \bar{\rho}^{1.55}
\end{aligned}
$$

In comparison, the scaling laws, as obtained by Khaderi et al. ${ }^{19}$, based on an idealised slender beam model of the gyroid lattice were $E=0.426 \bar{\rho}^{2} E_{S}, G=0.329 \bar{\rho}^{2} E_{S}$ and $\sigma_{Y S}=0.415 \bar{\rho}^{1.5} \sigma_{S}$. The pre-factor $C$ and exponent $n$ are in acceptable agreement between the two models. Differences are attributed to a lack of plateau borders in the slender beam model. The value of the exponents on $\bar{\rho}$ in the power-law scaling of $E^{*}, G^{\bullet}$ and $\sigma_{Y S}^{*}$ indicate that the lattice deforms mainly by bending and twisting of the struts under uniaxial strain and shear. This has also been observed in the macroscale compression of 3D printed gyroids ${ }^{19,34}$ and is associated with the low nodal connectivity of the gyroid lattice.

The effective mechanical properties of the hollow-wall gyroid lattice (as denoted by the superscript $\mathrm{o}$ ) are plotted in Fig. 10c,d. The following scaling relationships are noted for $0.02<\bar{\rho}<0.24$ :

$$
\begin{aligned}
& \frac{E^{\circ}}{E_{S}}=0.41 \bar{\rho}^{1.09} \\
& \frac{G^{\circ}}{E_{S}}=0.19 \bar{\rho}^{1.13} \\
& \frac{\sigma_{Y S}^{\circ}}{\sigma_{S}}=0.46 \bar{\rho}^{1.08}
\end{aligned}
$$

The magnitudes of the exponents for the hollow-wall gyroid lattice imply stretchingdominated behavior, attributed to the high shape efficiency of the tubular struts of the hollow-wall gyroid: the cell walls have a high bending stiffness and strength. These exponents are consistent with the measured compressive mechanical response of hollow-wall gyroid lattices at comparable relative densities ${ }^{35}$.

\subsection{Indentation response of solid-wall and hollow-wall gyroid coatings}

The contact width of the indenter is significantly greater than the unit cell size of the gyroids $(60 \mathrm{~nm})$ for the majority of recorded indentation depths in the nanoindentation 
experiments presented in Section 2.3. Hence, the discreteness of the gyroid microstructure plays a negligible role in the indentation response. Furthermore, previous analysis of the single gyroid lattice by Khaderi et al. ${ }^{19}$ has shown that both the elastic and plastic properties of the gyroid lattice are sufficiently isotropic that the Deshpande-Fleck isotropic foam model can be used to predict their mechanical response. The Deshpande-Fleck isotropic foam model has been previously used to successfully model the deformation response of nano-porous metals $s^{9,36,37}$ and is employed herein as a homogenised continuum model for the macroscopic deformation of the gyroid lattice during indentation. The main aspects of this model are outlined in the supplementary information section SI-5.

Indentation simulations are reported herein for a solid-wall gyroid of relative density $\bar{\rho}=0.40$ and for hollow-wall gyroid of $\bar{\rho}=0.14$ made from a parent material with Young's modulus $E$, Poisson's ratio $v_{S}=0.3$ and yield strength $\sigma_{Y S}$. From the effective material properties shown in Figure 10, it follows that, for the solid-wall gyroid, $E^{\bullet}=0.129 E_{S}{ }^{\bullet}, \sigma_{Y}^{\bullet}=0.114 \sigma_{Y S}{ }^{\bullet}$ and $\nu_{p}^{\bullet} \approx 0.32$. Similarly, for the hollow-wall gyroid, $E^{\circ}=0.048 E_{S}^{\circ}, \sigma_{Y}^{\circ}=0.056 \sigma_{Y S}{ }^{\circ}$ and $v_{p}^{\circ}=0.40$. Based on these values, the parent material modulus $E_{S}$ and strength $\sigma_{Y S}$ are treated as unknown parameters and are chosen to bring the simulated and measured indentation responses into good agreement for each coating thickness.

\subsection{Finite element modelling of indentation experiments}

Quasi-static finite strain indentation calculations were conducted using the commercial finite element package ABAQUS. A simplified axisymmetric model is used (Fig. 11) to model the indentation of each gyroid coating. To achieve this, the Berkovich indenter was modelled as a conical indenter of included semi-angle $\beta=70.3^{\circ}$ such that the nominal contact area $A_{C}$ is the same as that of the Berkovich tip for any indent depth ${ }^{38}$. The gyroid coating thickness adopts the values as measured by FIB. The glass substrate is modelled as a linear elastic solid, of thickness and radius $100 \mu \mathrm{m}$, possessing a modulus of $69.6 \mathrm{GPa}$ and Poisson ratio of 0.3. A $350 \mathrm{~nm}$ thick FTO layer is present between the gyroid coating and glass substrate, and this is treated as a linear elastic solid of modulus $150 \mathrm{GPa}$ and Poisson's ratio 0.3 as determined via a separate indentation test on an annealed FTO/glass substrate (see supplementary information Fig. SI-4). These values are consistent with previous indentation studies on thermally annealed FTO layers ${ }^{39}$ of similar thicknesses. It is assumed that the layers are ideally bonded together. The bottom of the mesh rests on a rigid, frictionless surface, with a symmetric boundary condition imposed on the centerline. The mesh was refined until the point was reached whereby a doubling of the mesh density led to less than $1 \%$ 
difference in the predicted indentation response (see supplementary information Fig. SI5.)

Contact between the indenter surface and gyroid coating was modelled using the Master-Slave surface contact algorithm within ABAQUS. The indenter was assumed to be rigid and frictionless, with a tip radius of $50 \mathrm{~nm}$, and was incrementally driven into the gyroid coating under successive loading and unloading cycles, with unloading after increments of indentation depth $\delta$ of $50 \mathrm{~nm}$. Preliminary calculations revealed that the Oliver and Pharr method gives an accurate measure for the true area of contact $A_{t}$ from the FE simulations, at any indentation depth $\delta$. Further, the true area of contact $A_{t}$ is within a few percent of the nominal area of contact $A_{c}=\pi \delta^{2} \tan ^{2} \beta$, with negligible pile-up or sink-in. Thus, it is adequate to define the hardness $H$ as the nominal contact pressure $H=P / A_{c}$ in the present study. The reduced modulus $E_{R}$ is obtained by fitting a power law to the unloading curve and subsequent use of the Sneddon equation as described in the supplementary information.

Predictions of the reduced modulus $E_{R}$ and hardness $H$ as a function of the normalised indentation depth $\delta / h$ are included in Figure 9. The measured and predicted indentation responses were brought into agreement across each coating thickness when the Young's modulus and uniaxial compressive strength of the solid-wall gyroid coating were ascribed the values of $E=25 \mathrm{GPa}$ and $\sigma_{Y S}=0.51 \mathrm{GPa}$. This implies that the apparent modulus of the parent nickel is $E_{S}=194 \mathrm{GPa}$ and the parent solid yield strength equals $\sigma_{Y S}=4.49 \mathrm{GPa}$. Hence, the inferred Young's modulus of the cell wall is consistent with that of electroplated nickel thin films ${ }^{40}$. However, the strength $\sigma_{Y S}$ of cell wall material exceeds the bulk value of pure nickel by more than an order of magnitude ${ }^{41}$. This result is consistent with previous findings of the inferred strutstrength of lattice materials with nanoscale strut diameters ${ }^{9,37}$.

For the hollow-wall gyroid lattice, the coating properties were found to be to $E=1.2$ $\mathrm{GPa}$ and $\sigma_{Y S}^{\circ}=0.055 \mathrm{GPa}$, implying parent material properties of $E_{S}=25.0 \mathrm{GPa}$ and $\sigma_{Y S}$ $=0.99 \mathrm{GPa}$. These values are significantly lower than the commonly quoted values of $E=$ $1060 \mathrm{GPa}$ and $\sigma_{Y S}=130 \mathrm{GPa}$ for monolayer graphene ${ }^{42}$. However, for $\delta / h>0.6$, the measured increase in $H$ and $E_{R}$ exceeds the predicted response for the foam model due to interaction with the hard FTO underlayer. To account for the significant reduction in the predicted stiffness and strength of the parent graphene material we seek an explanation at a lower length scale - that of the wavy walls of the hollow-wall graphitic struts. A detailed explanation is now provided.

\subsection{Effect of cell wall waviness on the in-plane cell wall modulus and strength}


The high-resolution TEM images such as Figure 8a reveal that a small degree of cell wall waviness is present (wavelength $\lambda \approx 5-15 \mathrm{~nm}$ ) in the cell wall of the hollow-wall gyroid lattice due to the relaxation of the $3 \mathrm{~nm}$ thick cell walls upon removal of the solid-wall nickel template. Upon loading, the walls of the gyroid lattice undergo stretching and bending; however the axial compliance of each cell wall is degraded by waviness. When the wavy stack of sheets is subjected to an axial strain, the misalignment induces longitudinal shear on the cross-section of the cell wall. Thus axial shortening is accommodated by longitudinal shear of the wavy stack of graphene layers. Consequently, the axial stiffness and axial strength of each cell wall is dictated by the out-of-plane shear properties of the few-layer graphene. This cell-wall deformation mode also dictates the axial compliance of wavy cell walls in graphitic foams ${ }^{26}$.

In order to determine whether this deformation mode is active in the few-layered hollow-wall graphene gyroid lattice, the relevant knockdown factors for stiffness and strength are obtained as follows, by treating the wavy cell wall as a beam of height $h$ and assume that an end tension $T$ induces longitudinal shear of the wavy beam, and thereby axial straining of the beam, as depicted in Fig. 8b (see supplementary information Fig. SI-3 for details). Inferred values of waviness amplitude $\omega_{0}<2.6 \mathrm{~nm}$ are obtained from both the cell wall modulus and strength values, as measured in the indentation tests. We conclude that a very small value of cell wall waviness is sufficient to knock-down the in-plane modulus and strength of the graphene cell-walls to the observed values.

\section{Solid-wall nickel gyroids and hollow-wall graphene gyroids in material property space}

A plot of compressive strength versus density is shown in Figure 1; it compares the properties of the gyroid lattices tested herein to other state-of-the-art lattice materials across a wide range of strut length-scales. The measured strength of solid-wall nickel gyroid rivals those of fully dense high strength $\mathrm{Ni}$ alloys, such as Inconel (highlighted in Fig. 1). This is consistent with other studies on nanoscale lattices such as nickel inverse opals $^{37}$ and for nickel double-gyroids ${ }^{9}$. Furthermore, the strength to density ratio of the bending-dominated solid-wall gyroid lattice exceeds that of the stretching-dominated micron-scale octet truss lattice, due to the size-strengthening effect present in the nanoscale gyroidal struts.

It is noted that the post-indentation recovery of the hollow-wall graphene gyroids from high levels of compressive strain is remarkably high, in contrast to macroscale 
templated graphene foams ${ }^{26}$. Lattice recoverability after compression is found in other low-density hollow lattices, owing to elastic buckling enabled by a size-strengthening effect ${ }^{44,45}$. Size-dependent effects can influence material properties beyond mechanical stiffness and strength, such as electrical conductivity. A plot of electrical conductivity versus density is shown in the supplementary information (Fig. SI-6), comparing the hollow-wall graphene gyroid against a range of graphene-based cellular materials. The measured conductivity of the hollow-wall graphene gyroid ${ }^{2}(80-90 \mathrm{~S} / \mathrm{cm})$ greatly exceeds that of other CVD-based foams $(10-17 \mathrm{~S} / \mathrm{cm})$. Conductivity is increased by more than an order of magnitude compared to materials based on flake assembly methods such as graphene aerogels $(0.01-2.5 \mathrm{~S} / \mathrm{cm})$. This combination of mechanical strength and recoverability with electrical conductivity demonstrates the size-effect enhanced functionality of the hollow-wall graphene gyroid lattice.

\section{Conclusions}

Indentation measurements and numerical simulations are reported to estimate the mechanical properties of a nickel solid-wall gyroid lattice and hollow-wall graphene gyroid lattice. Nickel gyroid coatings of thickness 300, 500 and $700 \mathrm{~nm}$, with unit cell sizes on the order of $60 \mathrm{~nm}$ and a relative density of $40 \%$, were manufactured by block copolymer self-assembly and electro-deposition of Ni. Hollow-wall graphene gyroid lattices were prepared through a CVD method using these nickel lattices as a template for the formation of few-layer graphene, resulting in a cell wall thickness of $3 \mathrm{~nm}$ corresponding to a relative density of approximately $14 \%$. Berkovich nano-indentation tests were then performed to determine both the hardness and modulus of the coatings.

Multi-scale finite element analysis was performed to extract both the effective mechanical properties of the gyroid coatings and the associated properties of the parent material. The solid-wall gyroid lattice undergoes a bending-dominated deformation mode, in contrast to the stretching-dominated hollow-wall gyroid lattice. Both gyroid coatings have high yield strength to density ratios, exceeding many other state-of-theart lattice materials at equal relative densities. This is attributed to the sizestrengthening effect present in nanoscale gyroid struts and walls. However, the cell-wall strength of the graphene gyroids is significantly less than the value for pristine monolayer graphene. This discrepancy is attributed to interlayer shear within the wavy walls of the few-layered graphene. The electrical conductivity of the hollow-wall gyroid lattice significantly exceeds that of other state-of-the-art graphene-based cellular materials. It is concluded that hollow-wall graphene gyroids combine size-dependent mechanical and electrical properties with a topology of high structural efficiency. These 
structures offer enhanced functionality for a wide range of emerging applications where high conductivity, high surface area, mechanical strength, and high yield strain are required.

\section{Acknowledgments}

We acknowledge funding from EPSRC (Grant No. EP/K016636/1, EP/P005152/1 and EP/P007767/1). K.N. acknowledges funding from the EPSRC Cambridge NanoDTC (Grant No. EP/G037221/1). V-P V-R further acknowledges support from NPL. BB and AJP acknowledge funding from the U.K. Department of Business, Energy and Industrial Strategy (NPL Project Number 121452). D. L. acknowledges funding from Clare College (Denman Baynes Senior Research Fellowship). 


\section{References}

1. Gibson, L. J. \& Ashby, M. F. The Mechanics of Three-Dimensional Cellular Materials. Proc. R. Soc. A Math. Phys. Eng. Sci. 382, 43-59 (1982).

2. Cebo, T. et al. Chemical vapour deposition of freestanding sub-60 $\mathrm{nm}$ graphene gyroids. Appl. Phys. Lett. 111, (2017).

3. Bauer, J., Schroer, A., Schwaiger, R. \& Kraft, O. Approaching theoretical strength in glassy carbon nanolattices. Nat. Mater. 15, 438-443 (2016).

4. Meza, L. R., Das, S. \& Greer, J. R. Strong, lightweight, and recoverable threedimensional ceramic nanolattices. Science (80-. ). 345, 1322-1326 (2014).

5. Gibson, L. J. \& Ashby, M. F. Cellular Solids: Structure and Properties. (Cambridge University Press, 1997).

6. Ronan, W., Deshpande, V. S. \& Fleck, N. A. The tensile ductility of cellular Solids: The role of imperfections. Int. J. Solids Struct. 102-103, 200-213 (2016).

7. Zheng, X. et al. Ultralight, ultrastiff mechanical metamaterials. Science (80-. ). 344, 1373-1377 (2014).

8. do Rosário, J. J. et al. Self-Assembled Ultra High Strength, Ultra Stiff Mechanical Metamaterials Based on Inverse Opals. Adv. Eng. Mater. 17, 1420-1424 (2015).

9. Khaderi, S. N. et al. The indentation response of Nickel nano double gyroid lattices. Extrem. Mech. Lett. 10, 15-23 (2017).

10. Arzt, E. Size effects in materials due to microstructural and dimensional constraints: a comparative review. Acta Mater. 46, 5611-5626 (1998).

11. Dresselhaus, M. S. et al. New directions for low-dimensional thermoelectric materials. Adv. Mater. 19, 1043-1053 (2007).

12. Kraft, O., Gruber, P. A., Mönig, R. \& Weygand, D. Plasticity in Confined Dimensions. Annu. Rev. Mater. Res. 40, 293-317 (2010).

13. Bauer, J., Schroer, A., Schwaiger, R. \& Kraft, O. Approaching theoretical strength in glassy carbon nanolattices. Nat. Mater. 15, 438-443 (2016).

14. Jang, D., Meza, L. R., Greer, F. \& Greer, J. R. Fabrication and deformation of threedimensional hollow ceramic nanostructures. Nat. Mater. 12, 893-898 (2013).

15. Gibson, L. J. Biomechanics of cellular solids. J. Biomech. 38, 377-399 (2005).

16. Gan, Z., Turner, M. D. \& Gu, M. Biomimetic gyroid nanostructures exceeding their natural origins. Sci. Adv. 2, 4-10 (2016).

17. Hsueh, H.-Y. \& Ho, R.-M. Bicontinuous ceramics with high surface area from block copolymer templates. Langmuir 28, 8518-29 (2012). 
18. Crossland, E. J. W. et al. A bicontinuous double gyroid hybrid solar cell. Nano Lett. 9, 2807-2812 (2009).

19. Khaderi, S. N., Deshpande, V. S. \& Fleck, N. A. The stiffness and strength of the gyroid lattice. Int. J. Solids Struct. 51, 3866-3877 (2014).

20. Owens, J. R., Daniels, C., Nicolaï, A., Terrones, H. \& Meunier, V. Structural, energetic, and electronic properties of gyroidal graphene nanostructures. Carbon N. Y. 96, 998-1007 (2016).

21. Jung, G. S. \& Buehler, M. J. Multiscale Mechanics of Triply Periodic Minimal Surfaces of Three-Dimensional Graphene Foams. Nano Lett. 18, 4845-4853 (2018).

22. Qin, Z., Jung, G. S., Kang, M. J. \& Buehler, M. J. The mechanics and design of a lightweight three-dimensional graphene assembly. Sci. Adv. 3, e1601536 (2017).

23. Miller, D. C., Terrones, M. \& Terrones, H. Mechanical properties of hypothetical graphene foams: Giant Schwarzites. Carbon N. Y. 96, 1191-1199 (2016).

24. Chen, Z. et al. Three-dimensional flexible and conductive interconnected graphene networks grown by chemical vapour deposition. Nat. Mater. 10, 424428 (2011).

25. Liu, Z. et al. Observation of microscale superlubricity in graphite. Phys. Rev. Lett. 108, (2012).

26. Nakanishi, K. et al. Compressive behavior and failure mechanisms of freestanding and composite 3D graphitic foams. Acta Mater. 159, 187-196 (2018).

27. Dolan, J. A. et al. Gyroid Optical Metamaterials: Calculating the Effective Permittivity of Multidomain Samples. ACS Photonics 3, 1888-1896 (2016).

28. Pimenta, M. A. et al. Studying disorder in graphite-based systems by Raman spectroscopy. Phys. Chem. Chem. Phys. 9, 1276-1290 (2007).

29. Ning, G. et al. Gram-scale synthesis of nanomesh graphene with high surface area and its application in supercapacitor electrodes. Chem. Commun. (Camb). 47, 5976-5978 (2011).

30. Oliver, W. C. \& Pharr, G. M. An improved technique for determining hardness and elastic modulus using load and displacement sensing indentation experiments. $J$. Mater. Res. 7, 1564-1583 (1992).

31. Lambert, C. A. et al. Triply periodic level surfaces as models for cubic tricontinuous block copolymer morphologies. Philos. Trans. R. Soc. London. Ser. A Math. Phys. Eng. Sci. 354, 2009-2023 (1996).

32. Pingle, S. M., Fleck, N. A., Deshpande, V. S. \& Wadley, H. N. G. Collapse mechanism maps for a hollow pyramidal lattice. Proc. R. Soc. A Math. Phys. Eng. 
Sci. 467, 985-1011 (2011).

33. Omairey, S. L., Dunning, P. D. \& Sriramula, S. Development of an ABAQUS plugin tool for periodic RVE homogenisation. Eng. Comput. 0, 1-11 (2018).

34. Maskery, I. et al. Insights into the mechanical properties of several triply periodic minimal surface lattice structures made by polymer additive manufacturing. Polymer (Guildf). 152, 62-71 (2018).

35. Al-Ketan, O. et al. Microarchitected Stretching-Dominated Mechanical Metamaterials with Minimal Surface Topologies. Adv. Eng. Mater. 20, 1800029 (2018).

36. Lührs, L., Soyarslan, C., Markmann, J., Bargmann, S. \& Weissmüller, J. Elastic and plastic Poisson's ratios of nanoporous gold. Scr. Mater. 110, 65-69 (2016).

37. Pikul, J. H. et al. High strength metallic wood from nanostructured nickel inverse opal materials. Sci. Rep. 9, 719 (2019).

38. Min, L., Wei-min, C., Nai-gang, L. \& Ling-Dong, W. A numerical study of indentation using indenters of different geometry. J. Mater. Res. 19, 73-78 (2005).

39. Gao, Q. et al. Improved mechanical properties of SnO2:F thin film by structural modification. Ceram. Int. 40, 2557-2564 (2014).

40. Luo, J. K., Flewitt, A. J., Spearing, S. M., Fleck, N. A. \& Milne, W. I. Young's modulus of electroplated Ni thin film for MEMS applications. Mater. Lett. 58, 2306-2309 (2004).

41. El-Sherik, A. M., Erb, U., Palumbo, G. \& Aust, K. T. Deviations from hall-petch behaviour in as-prepared nanocrystalline nickel. Scr. Metall. Mater. 27, 11851188 (1992).

42. Lee, C., Wei, X., Kysar, J. W. \& Hone, J. Measurement of the Elastic Properties and Intrinsic Strength of Monolayer Graphene. Science (80-. ). 321, 385-388 (2008).

43. Hodge, A. M. et al. Scaling equation for yield strength of nanoporous open-cell foams. Acta Mater. 55, 1343-1349 (2007).

44. Salari-Sharif, L., Valdevit, L. \& Schaedler, T. A. Energy dissipation mechanisms in hollow metallic microlattices. J. Mater. Res. 29, 1755-1770 (2014).

45. Meza, L. R., Das, S. \& Greer, J. R. Strong, Lightweight and Recoverable Three Dimensional Ceramic Nanolattices. Submitted 345, 1322-1326 (2014). 
Figures

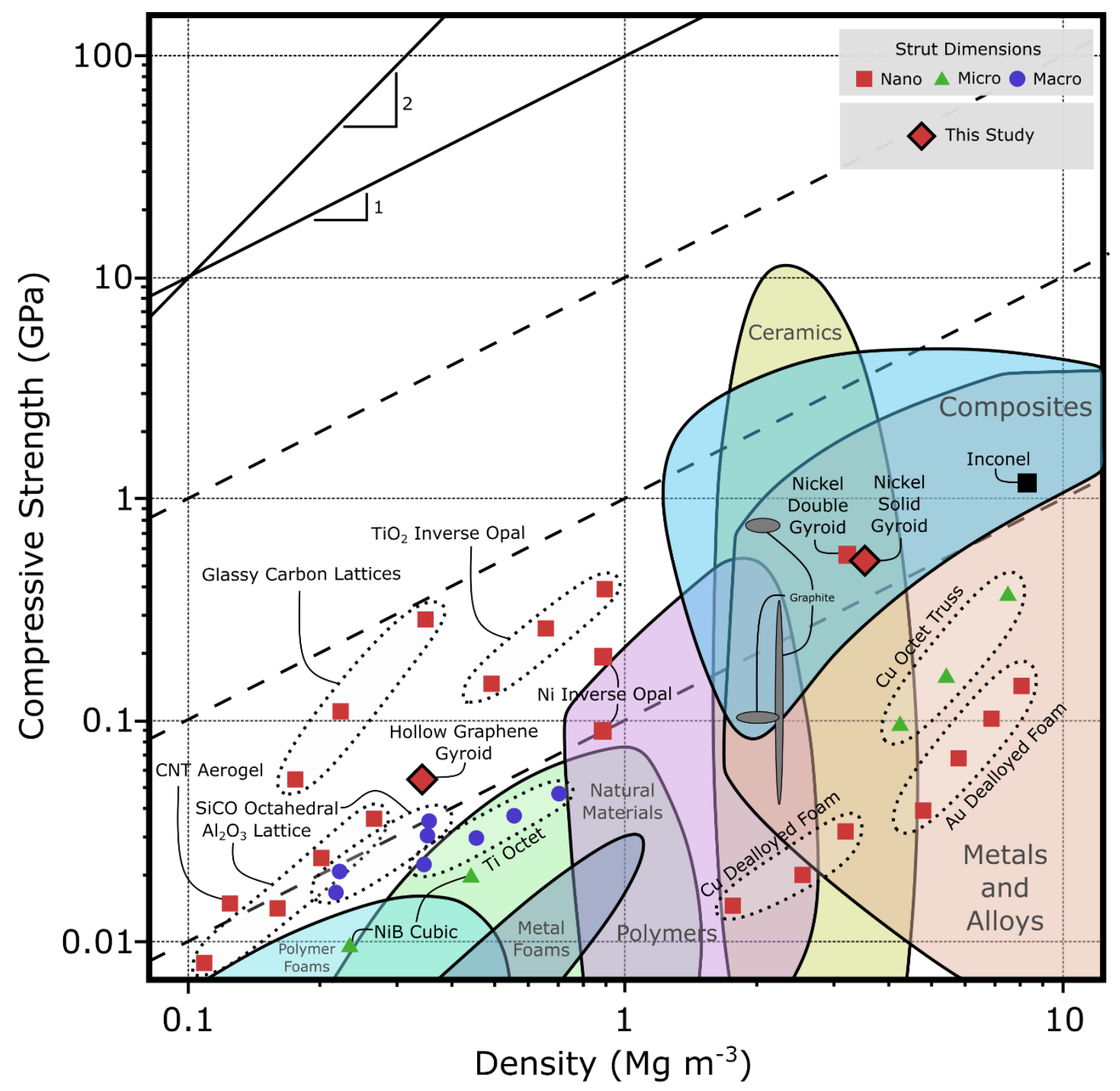

Figure 1. Chart of compressive strength versus density including the solid-wall nickel and hollow-wall graphene gyroids, as measured in the present study. Other state-of-theart lattice materials are highlighted according to the length scale of their cellular architecture. 


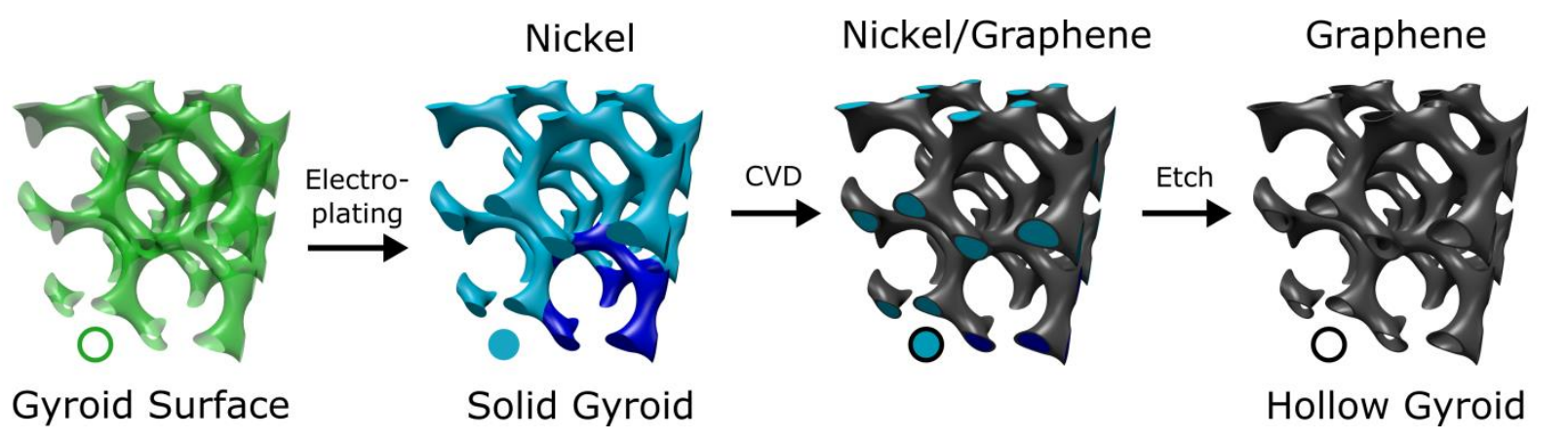

Figure 2. Schematic of the templated growth of the hollow-wall gyroid from a solid-wall gyroid. Sections shown are $2 \times 2 \times 2$ segments of the gyroid lattice, with a unit cell highlighted. Solid-wall nickel gyroids are prepared from polymeric templates using electroplating. A few-layer graphene film is grown on the nickel gyroid using CVD and isolated with wet chemical etching to generate the hollow-wall graphene gyroid.
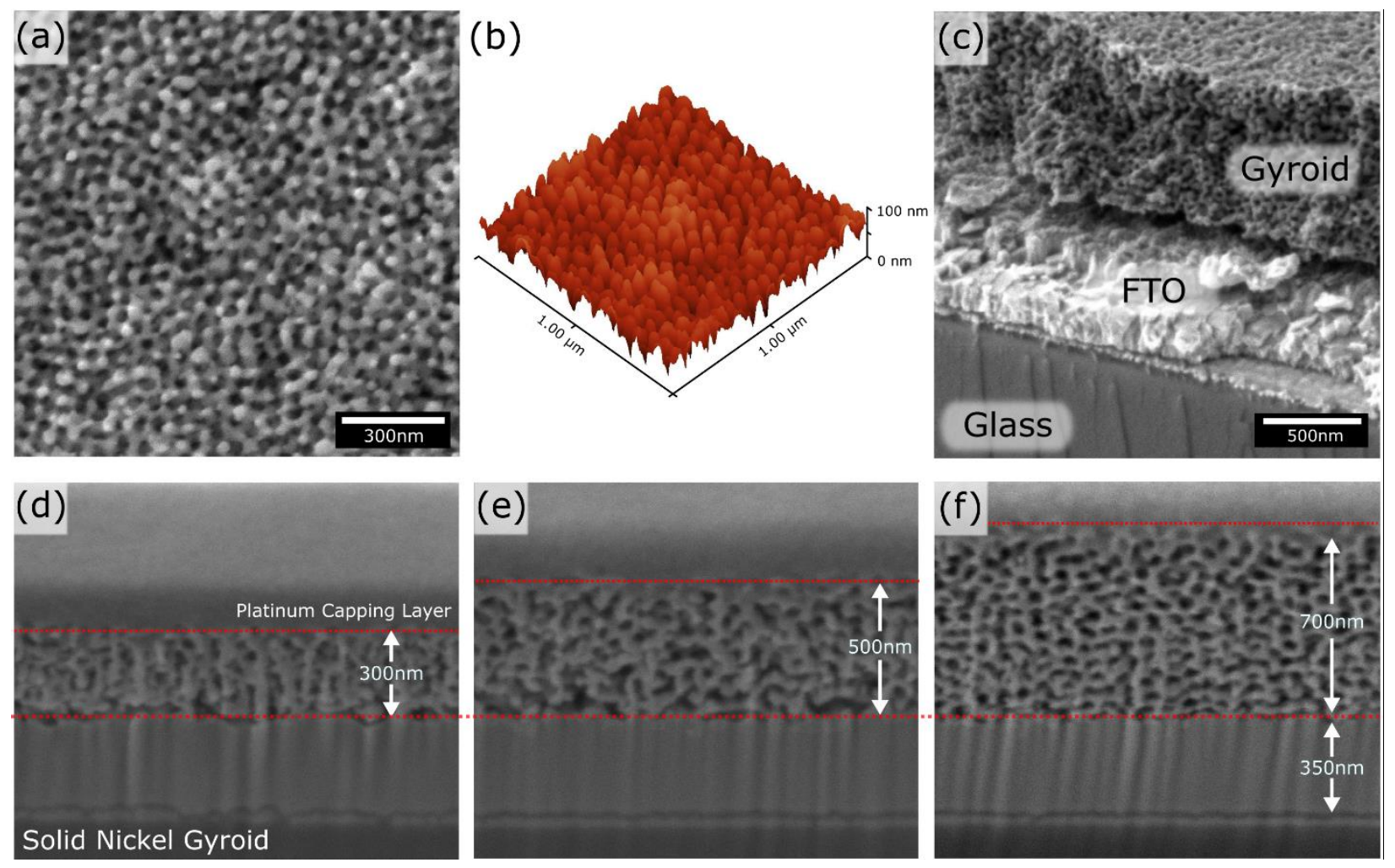

Figure 3. SEM and AFM micro-graphs of the nickel solid-wall gyroid coating showing $(a, b)$ the top surface of the coating, and (c) a cleaved cross-sectional image in which the gyroid, FTO and glass layers are clearly visible. Cross-sectional SEM display the nickel solid-wall gyroid coating across three thicknesses (d) $300 \mathrm{~nm}$ (e) $500 \mathrm{~nm}$ and (f) $700 \mathrm{~nm}$. The capping layer is platinum, deposited as a part of the FIB milling process. 

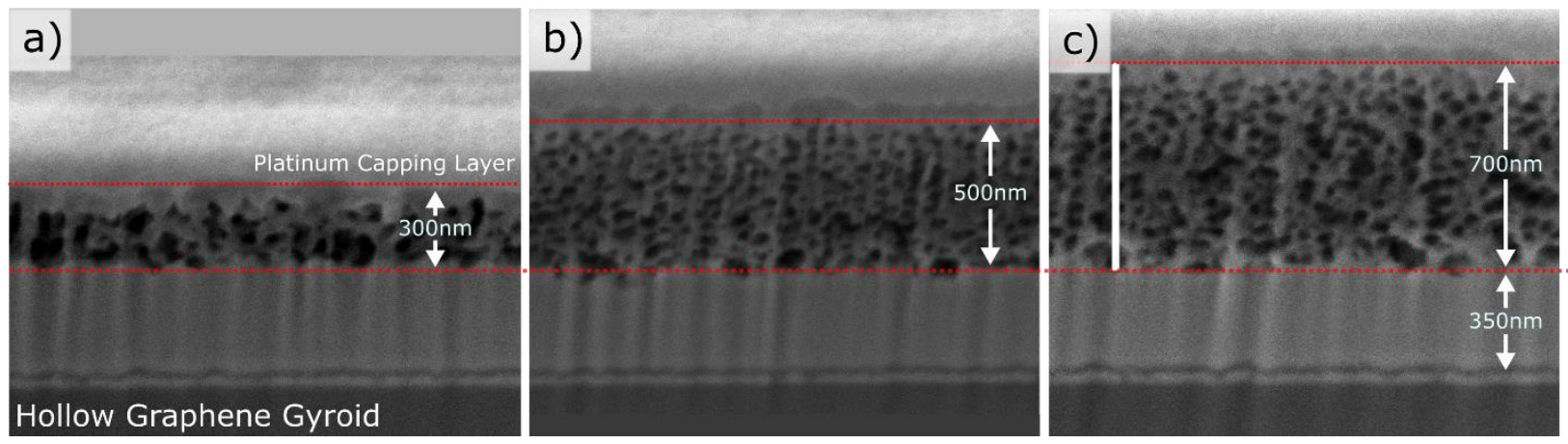

Figure 4. Cross-sectional SEM micro-graphs showing the templated growth of freestanding hollow-wall graphene gyroid coating across three thicknesses (a) $300 \mathrm{~nm}$ (b) $500 \mathrm{~nm}$ and (c) $700 \mathrm{~nm}$. The capping layer is platinum, deposited as a part of the FIB milling process.
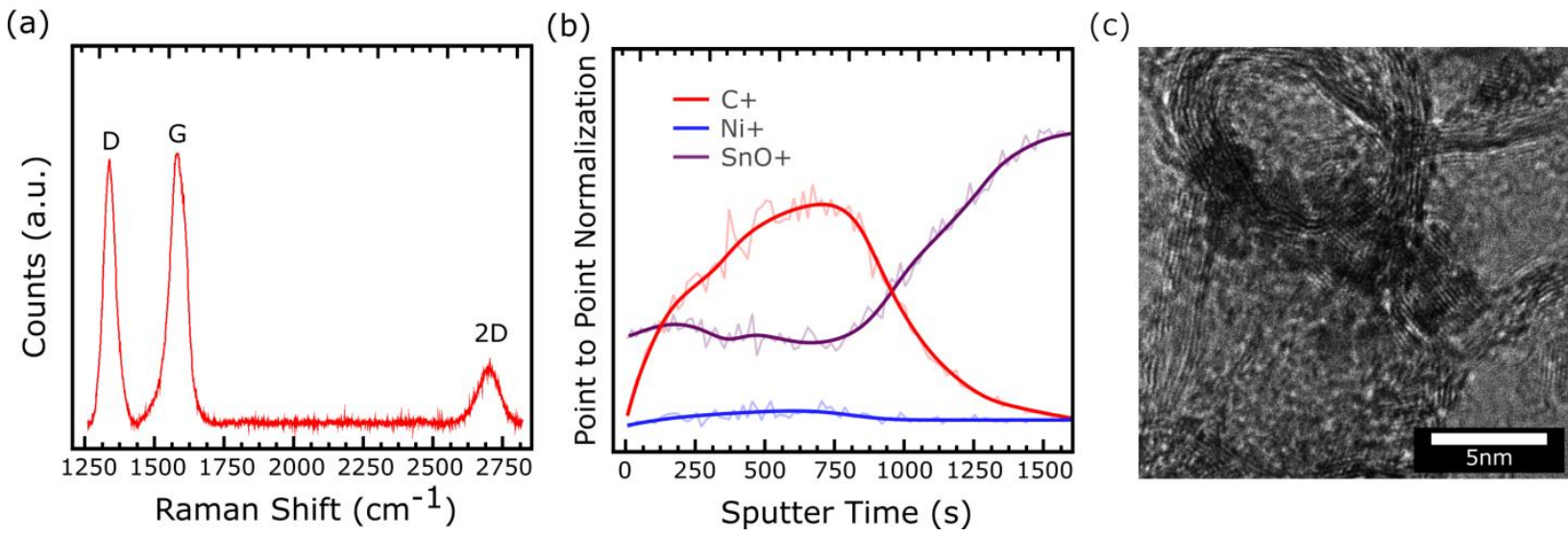

Figure 5. (a)Representative Raman spectra of the hollow-wall graphene gyroid surface. Prominent D, $G$ and 2D peaks are present, characteristic of few-layer graphene. (b) ToFSIMS depth profile of the hollow-wall graphene gyroid lattice. A negligible $\mathrm{Ni}^{+}$signal is present throughout the bulk of the lattice, confirming removal of the internal template after etching. (c) HR-TEM image of a hollow-wall graphene gyroid channel with diameter of $\sim 15 \mathrm{~nm}$. 


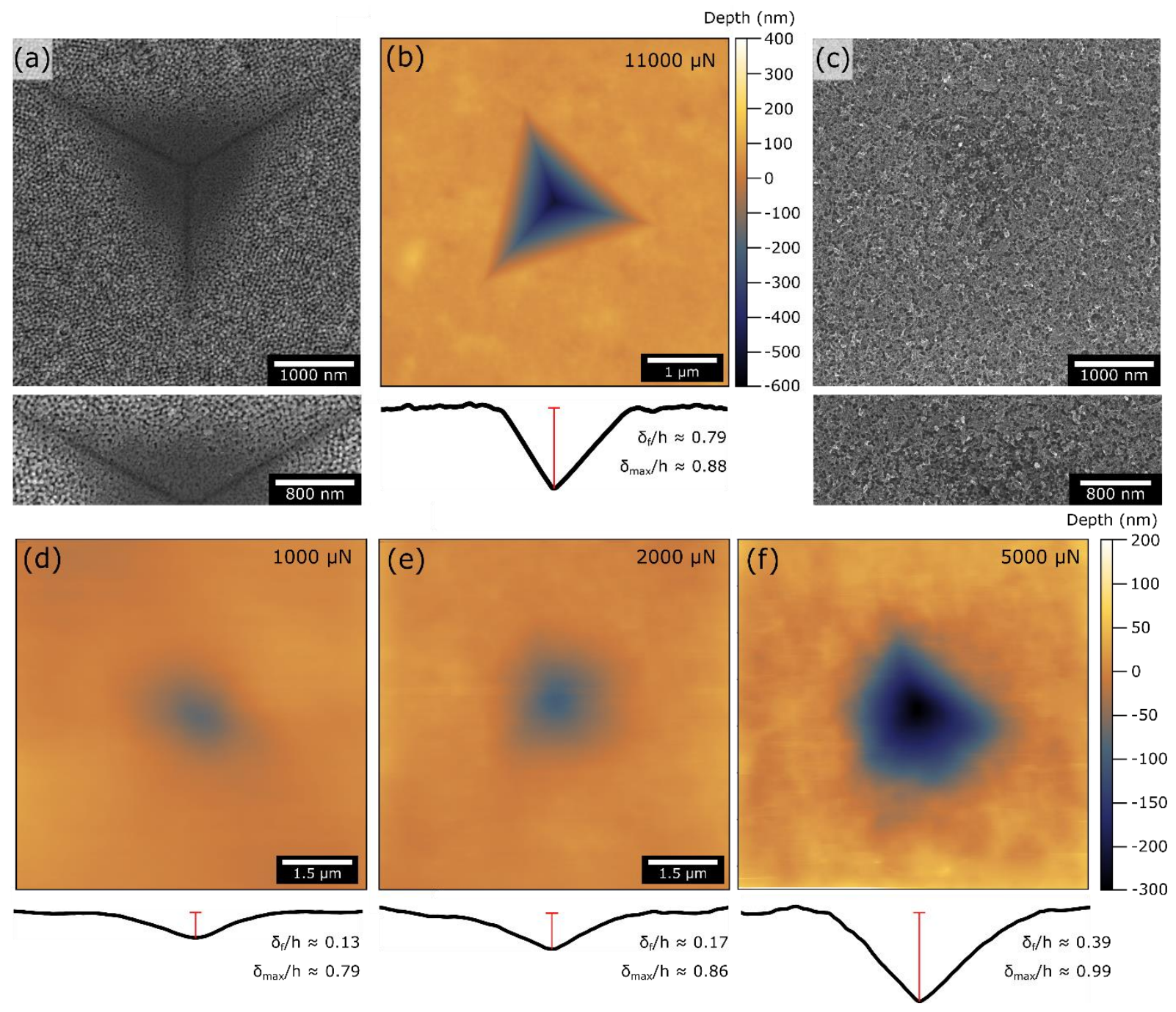

Figure 6. SEM and surface micrographs of the solid-wall nickel gyroid and hollow-wall graphene gyroid taken post-indentation. $(a, b)$ A clear impression of the Berkovich tip is present in the nickel solid-wall gyroid lattice after indentation, with minimal elastic recovery. (c) Surface SEM image of the hollow-wall graphene gyroid displaying minimal damage after indentation to $\delta_{\max } / \mathrm{h} \approx 0.79$. Surface profiles after progressively greater indentation loading (d) $1000 \mu \mathrm{N}$, (e) $2000 \mu \mathrm{N}$, (f) $5000 \mu \mathrm{N}$ was applied to the hollow-wall graphene gyroid lattice. Significant elastic recovery is observed in all cases. 


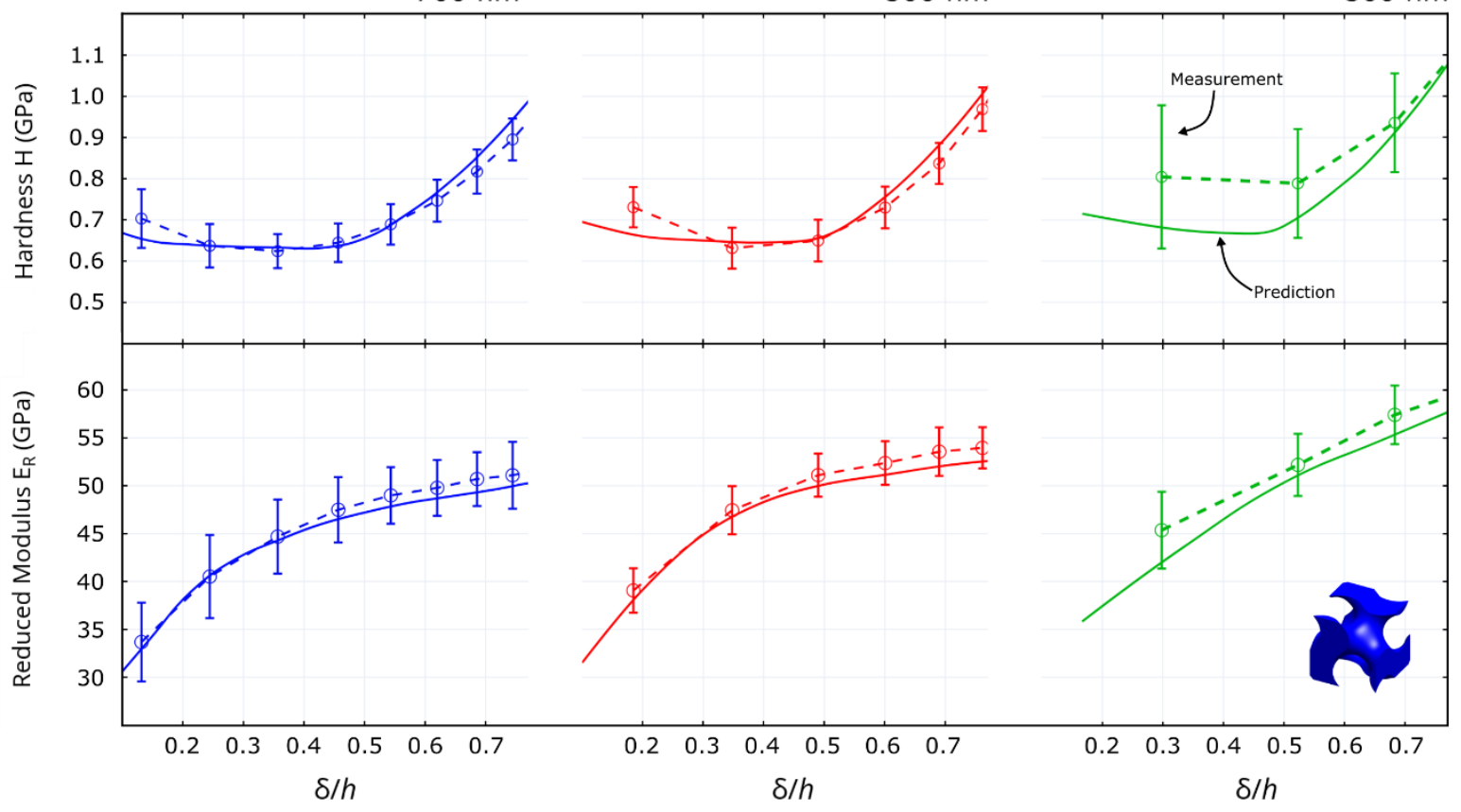

(b) Hollow Graphene Gyroid $700 \mathrm{~nm}$

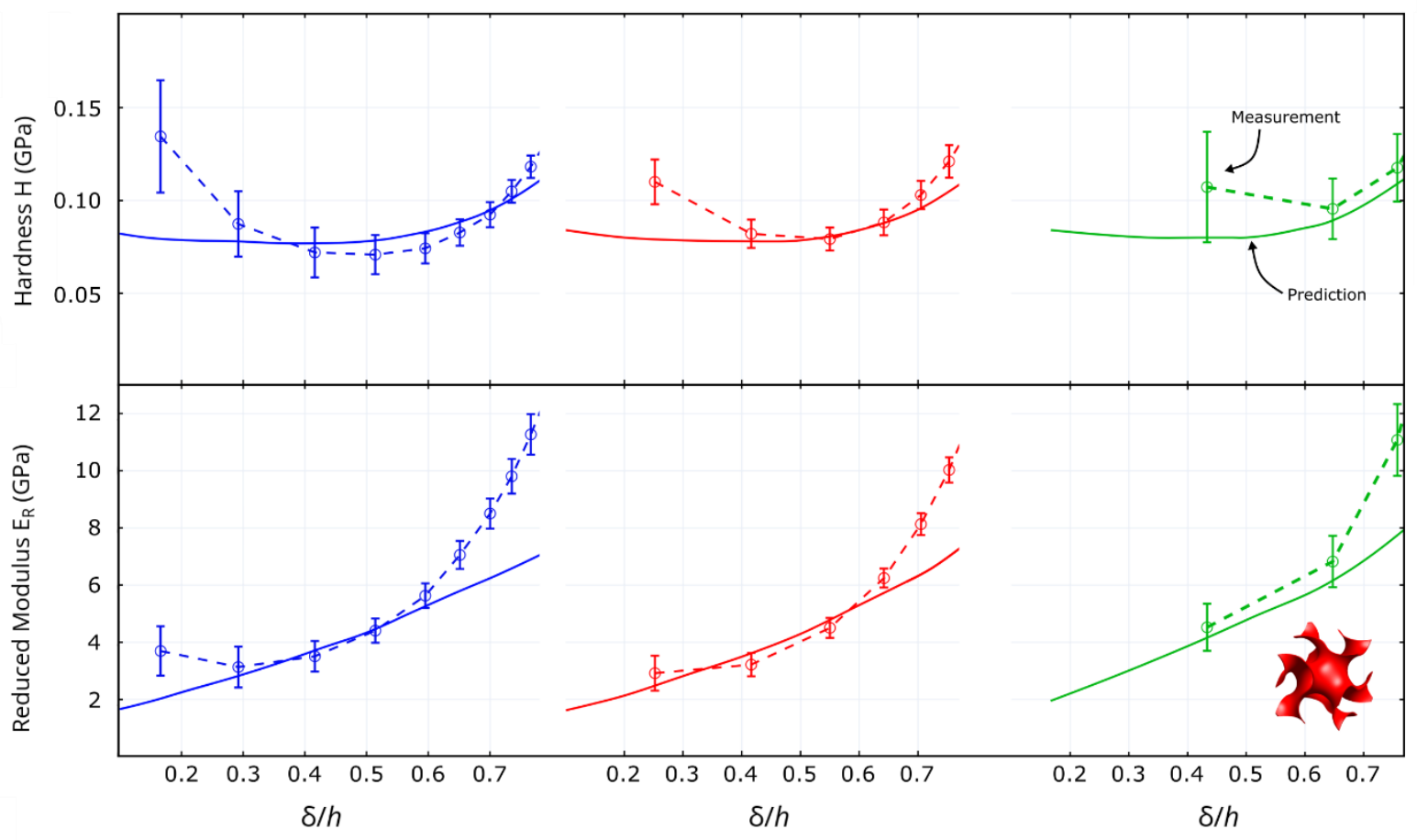

Figure 7. Hardness $\mathrm{H}$ and reduced modulus $\mathrm{E}_{\mathrm{R}}$ of the (a) solid-wall and (b) hollow-wall gyroid coatings as a function of the normalised indentation depth $\delta / h$. The error bars indicate the standard deviation over the 16 tests conducted on each coating. The FE predictions with nickel solid-wall gyroid coating properties $\mathrm{E}^{\bullet}=25 \mathrm{GPa}, v^{\bullet}=0.3$ and 
$\sigma_{\mathrm{Y}}^{\circ}=510 \mathrm{MPa}$ and hollow-wall graphene gyroid coating properties $\mathrm{E}^{\circ}=1.2 \mathrm{GPa}, v^{\circ}=$ 0.32 and $\sigma_{\mathrm{Y}}^{\circ}=55 \mathrm{MPa}$ are included. 


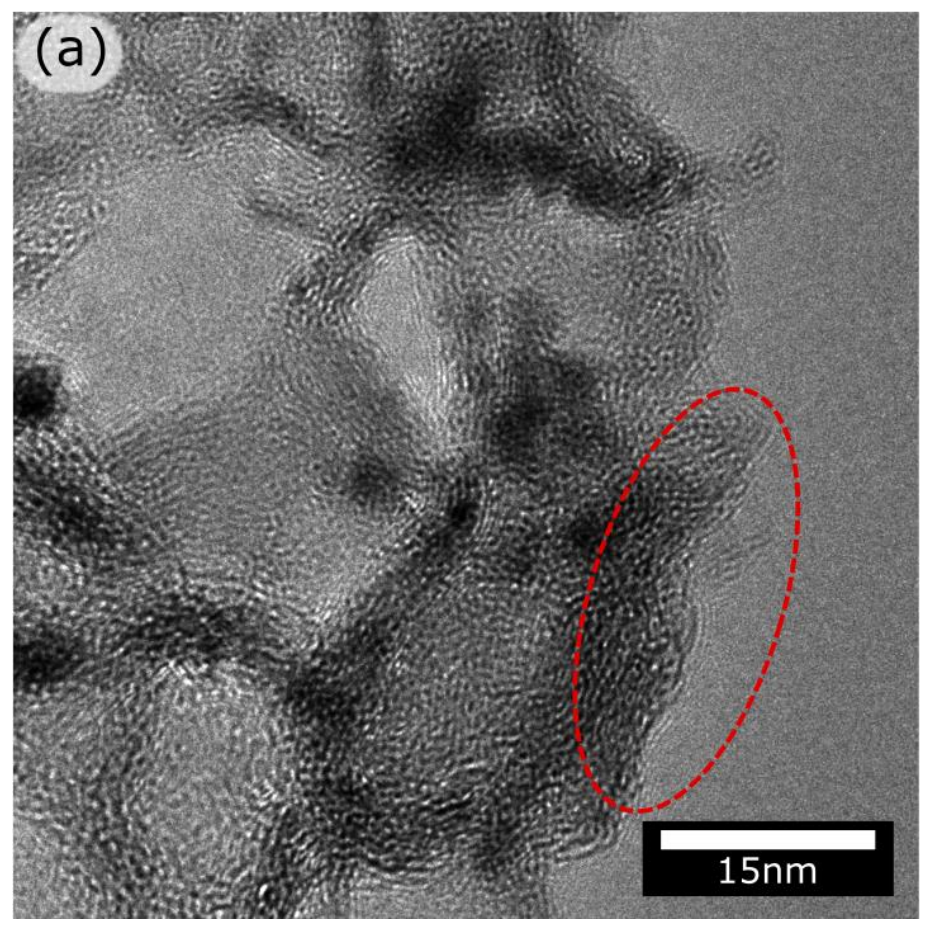

(b)

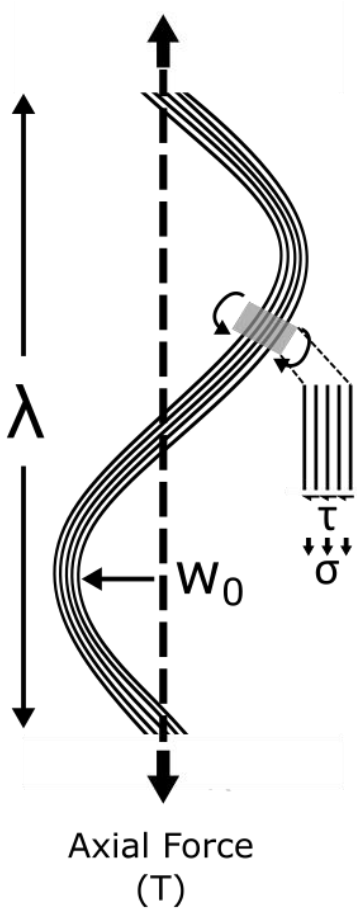

Figure 8. (a) HR-TEM image of the edges of a hollow-wall graphene gyroid showing the waviness of the few-layer graphene structure. (b) Wall level schematic idealising the waviness as a sine wave of amplitude $w_{0}$ and wavelength $\lambda$. In a wavy wall subjected to an axial tension, misalignment induces transverse shear forces on the cross-section of the cell wall, leading to interlayer shearing.
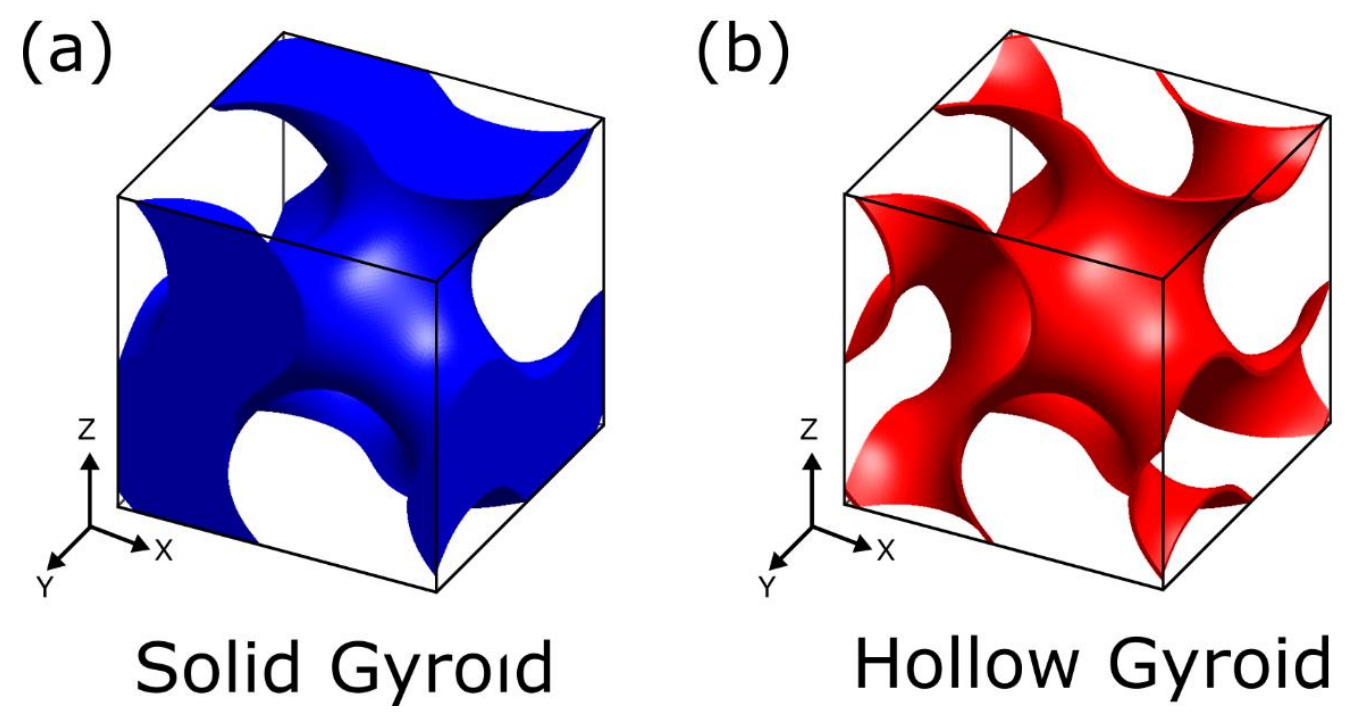

Figure 9. Representative volume elements of the solid-wall gyroid and hollow-wall gyroid lattices. In this sketch, a solid-wall gyroid with $\bar{\rho}=0.40$ and hollow-wall gyroid with $\bar{\rho}=0.14$ are shown. 
(a)

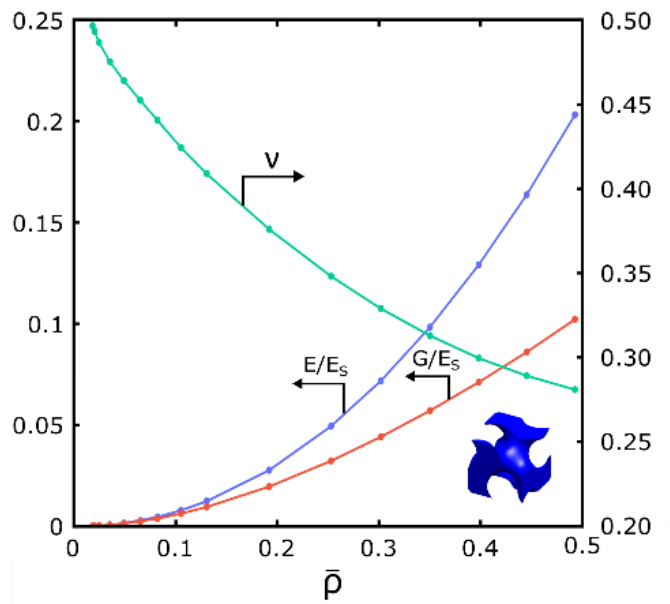

(c)

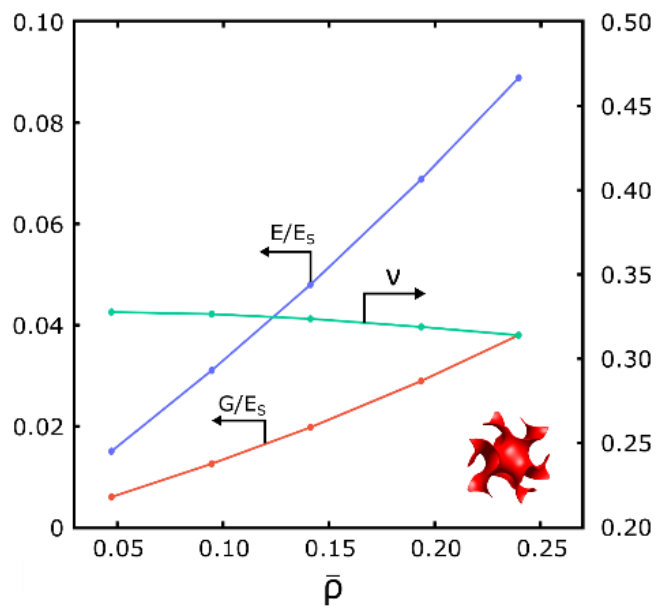

(b)

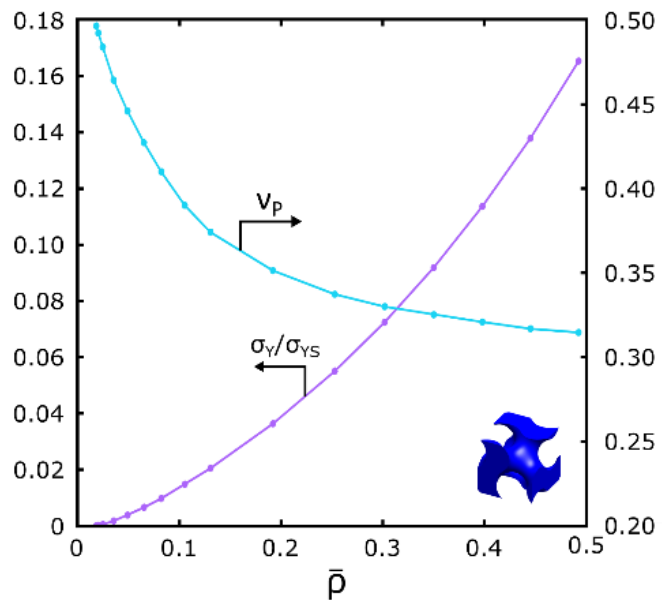

(d)

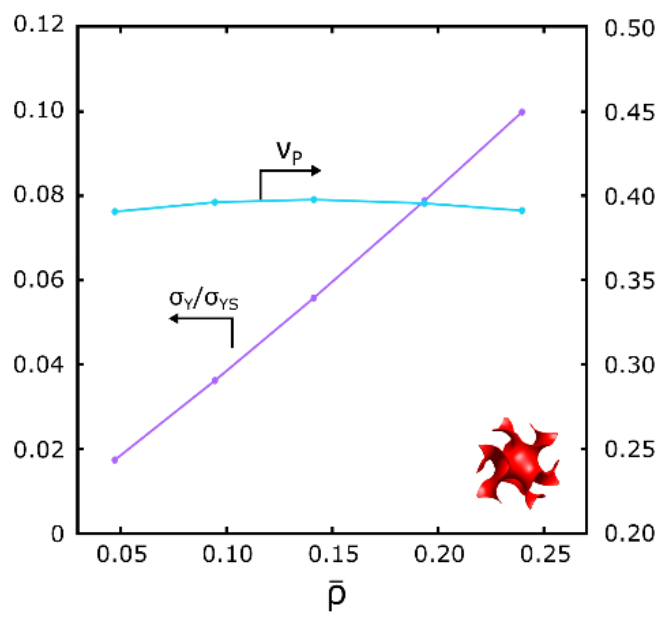

Figure 10. FE predictions of the effective mechanical properties of the solid-wall gyroid and hollow-wall gyroid lattices, made from isotropic elastic-perfectly plastic materials. The variation of normalised moduli $E / E_{S}, G / E_{s}$ and $v$ with relative density $\bar{\rho}$ for (a) the solid-wall gyroid and (c) the hollow-wall gyroid display different scaling behavior. A similar comparison can be made for the variation of $\sigma_{\mathrm{YS}} / \sigma_{\mathrm{S}}$ and $v_{p}$ with $\bar{\rho}$ for the (b) solid-wall gyroid and (d) hollow-wall gyroid. 


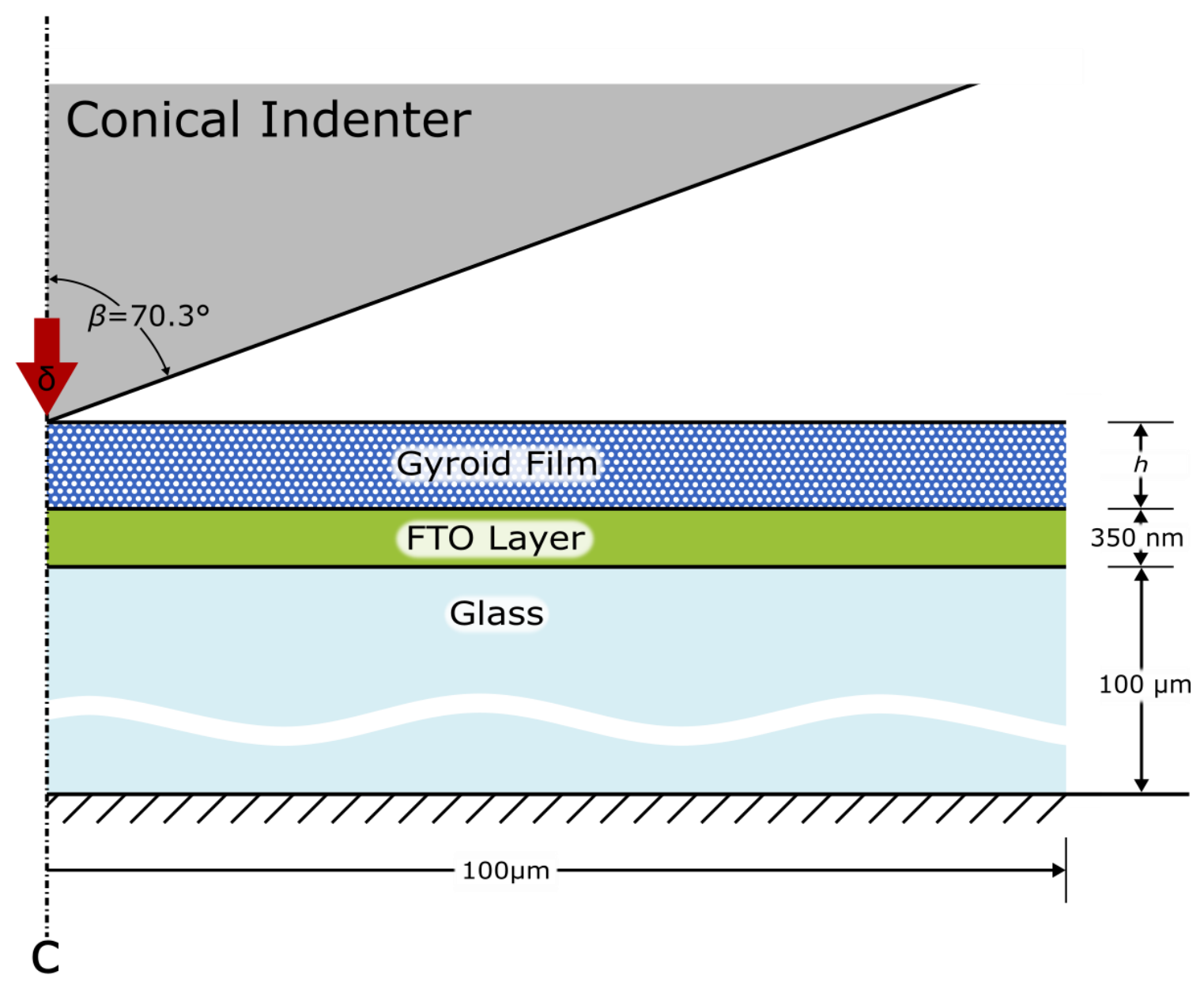

Figure 11. Schematic of the axisymmetric conical indentation model used to simulate the Berkovich nanoindentation response of gyroid coatings on a FTO/glass substrate. 